\title{
Soluble Graphene Through Edge-Selective Functionalization
}

\author{
Zhengzong Sun, Shin-ichiro Kohama, Zengxing Zhang, Jay R. Lomeda, and James M. Tour ( $\varangle)$ \\ Department of Chemistry and Mechanical Engineering and Materials Science and the Smalley Institute for Nanoscale Science and \\ Technology, Rice University, MS 222, 6100 Main Street, Houston, Texas 77005, USA \\ Received: 8 December 2009 / Revised: 18 December 2009 / Accepted: 22 December 2009 \\ (C) The Author(s) 2010. This article is published with open access at Springerlink.com
}

\begin{abstract}
Thermally expanded graphite was functionalized with 4-bromophenyl addends using the in situ diazonium formation procedure, and after mild sonication treatment in $N, N^{\prime}$-dimethylformamide, thin graphene layers were exfoliated from the bulk graphite. These chemically-assisted exfoliated graphene (CEG) sheets had higher solubility than pristine graphene without any stabilizer additive. More than $70 \%$ of these soluble flakes had less than 5 layers. Energy filtered transmission electron microscopy (EFTEM) elemental mapping provided evidence of the edge-selective diazonium functionalization with graphene. A majority of the Br signals came from the edges of the CEG indicating that the basal planes were not highly functionalized. The CEG was also characterized by $\mathrm{X}$-ray photoelectron spectroscopy, atomic force microscopy, Raman spectroscopy, and transmission electron microscopy.
\end{abstract}

\section{KEYWORDS}

Edge-selective functionalization, graphene, energy filtered transmission electron microscopy (EFTEM), chemicallyassisted exfoliation

\section{Introduction}

Graphene is defined as sheets of sp2-hybridized carbon, where the number of stacked sheets is $<10$ [1]. The production of chemically converted graphene from the reduction of graphene oxide is a convenient method to obtain large amounts of graphene [2-4]; however, even with efficient reducing agents such as hydrazine or $\mathrm{H}_{2}$, and annealing at high temperature, the original crystalline structure of graphene is not restored. Graphene oxide is heavily functionalized with many permanent chemical defects, such as holes, introduced into the basal plane. These holes are not readily healed even upon annealing.

Micromechanically cleaved graphene affords crystalline single sheets of graphene using highly ordered pyrolytic graphite and adhesive tape [5]. While this method opened an avenue for many fundamental studies, it is likely impossible to scale for the production of larger quantities.

The exfoliation of graphene in the liquid phase, by continuously sonicating graphite in a high surfacetension organic solvent [6,7], is another route to obtain mono-layered or few-layered sheets of graphene that are suspended in a dark mixture. However, the suspended graphene sheets tend to aggregate due to the lack of stabilizing groups such as surfactants or polymers to reduce interlayer attractions [8]. Because of significant $\pi-\pi$ interactions, unlike graphene oxide and chemically converted graphene, liquid

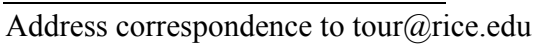


phase exfoliated graphene cannot be stabilized as a concentrated mixture.

Chemical vapor deposition (CVD) techniques have been used to grow thin and highly crystalline graphitic layers atop catalysts $[9,10]$. At elevated temperatures, methane or other volatile carbon precursors are decomposed on metal catalysts, such as $\mathrm{Ni}$ or $\mathrm{Cu}$, in a reductive atmosphere to produce graphene [11]. Epitaxial growth by annealing $\mathrm{SiC}$ at temperatures as high as $2000{ }^{\circ} \mathrm{C}$ produces graphene that is deposited directly on the SiC wafer [12-14]. This method is referred to as chemical solid deposition (CSD) since the precursor is a solid rather than a gas. While these growth methods, coupled with patterning, will likely be among the dominant growth methods for future high-performance graphene electronics, generating bulk scales of few-layered graphene for other materials applications will be problematic using the CVD and CSD approaches.

Recently, chemically converted graphene was successfully functionalized using diazonium salts and the product showed improved solubility in polar aprotic organic solvents compared to unfunctionalized chemically converted graphene [15]. In this paper, thermally expanded graphite is predominantly edge-functionalized with 4-bromophenyl groups using in situ formation of the corresponding diazonium salt from 4-bromoaniline. Mild sonication in $N, N^{\prime}$-dimethylformamide (DMF) then exfoliates thin chemically-assisted exfoliated graphene (CEG) sheets from the bulk functionalized graphite. CEG is more soluble than pristine graphene in DMF. In addition, more than $70 \%$ of the CEG flakes have less than 5 layers. The edge functional groups are detected by elemental mapping using energy filtered transmission electron microscopy (EFTEM). By taking advantage of the edge-selective functionalization, and low basal plane disruption, a bulk preparation of soluble yet more conductive graphene has been demonstrated.

\section{Results and discussion}

\subsection{Protocol for chemical-assisted exfoliation}

Scheme 1 shows the experimental procedure for making CEG. Expanded graphite was first dispersed in chlorosulfonic acid, showing a relatively high solubility of $0.97 \mathrm{mg} / \mathrm{mL}$. The diazonium salt was formed in situ from 4-bromoaniline in the presence of sodium nitrite and a catalytic amount of azobisisobutyronitrile (AIBN) and 4-bromophenyl groups were grafted mainly on the exposed edges of the expanded graphite flakes to produce functionalized expanded graphite. Because the diazonium salt of 4-bromoaniline is a relatively bulky molecule, it does not penetrate and react to the same degree with all of the graphene surfaces. In addition, the edges of the expanded graphite are more accessible than the interior basal plane surfaces that are stacked with strong $\pi-\pi$ interactions. After $1 \mathrm{~h}$ of bath sonication of the functionalized expanded graphite flakes in DMF and $1 \mathrm{~h}$ of centrifugation at $3200 \mathrm{r} / \mathrm{min}$, the CEG was collected from the supernatant. The functionalized CEG was much more soluble in DMF than the expanded graphite, as shown in Fig. 1.

As shown in the inset to Fig. 1, expanded graphite and CEG were sonicated separately (ultrasonic cleaner Cole-Parmer model 08849-00) for $1 \mathrm{~h}$ in DMF, followed by centrifugation for $1 \mathrm{~h}$ at $3200 \mathrm{r} / \mathrm{min}$ (Adams Analytical centrifuge CT-3201) and the supernatant was decanted

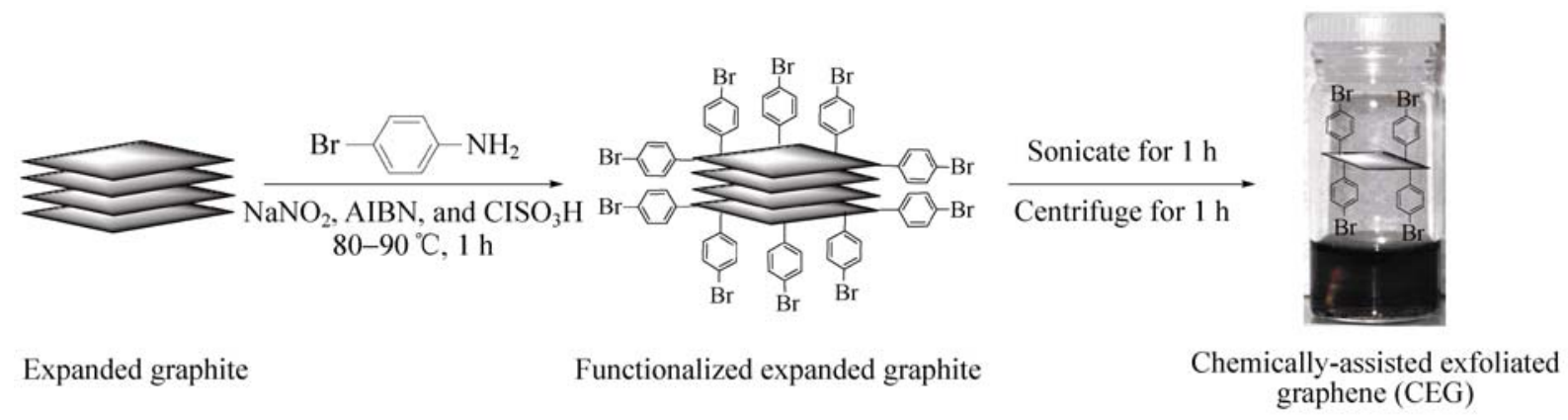

Scheme 1 Production of chemically-assisted exfoliated graphene (CEG) 
from the solid. The supernatant on the right in the inset to Fig. 1 is the expanded graphite control while the vial on the left holds the CEG supernatant; it has a much darker color and has remained stable for more than 3 months, indicating that the CEG is soluble in DMF while expanded graphite is poorly, if at all, soluble. The presumed CEG structure is shown in Fig. 1 with functional groups around the edges.

\subsection{Functionality and quality of graphene films}

The CEG was analyzed by X-ray photoelectron spectroscopy (XPS), as shown in Fig. 2. The XPS spectrum had a $\mathrm{Br} 3 \mathrm{~d}$ peak at $71 \mathrm{eV}$; unlike 4-bromophenyl diazonium functionalized chemically converted graphite $(3.2 \% \mathrm{Br})$ [15], much less bromine was detected in the CEG $(0.4 \%)$. Also, the sharp and symmetric $\mathrm{C} 1 \mathrm{~s}$ peak at $285 \mathrm{eV}$ confirmed the presence of a graphitic structure with minimal oxidation, indicating that a large number of crystalline domains remained after the reaction. Energy dispersive X-ray spectroscopy (EDX) was used as a supplemental technique to investigate the $\mathrm{Br}$ concentration on single-sheet CEG flakes. The Br concentration varied from $0.56 \%$ to $0.67 \%$, values that are comparable to the values obtained by XPS.

The crystalline structure of the CEG was confirmed

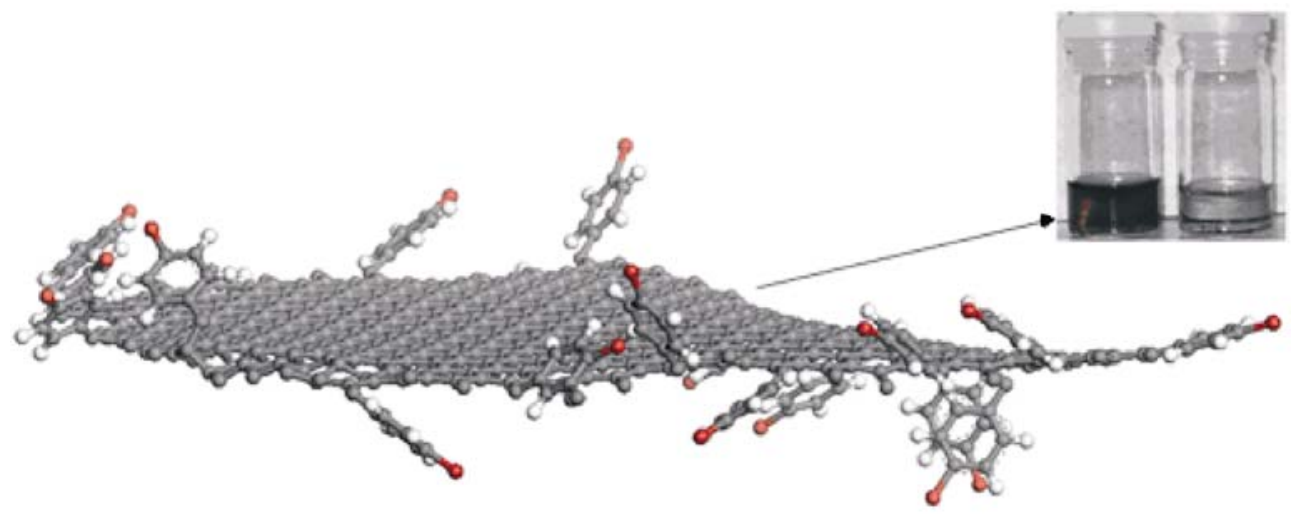

Figure 1 CEG structure (gray: $\mathrm{C}$ atoms; red: $\mathrm{Br}$ atoms; white: $\mathrm{H}$ atoms). The inset shows the expanded grpahite control supernatant (right vial) and the CEG supernatant (left vial) after both had been treated by sonication and centrifugation in DMF

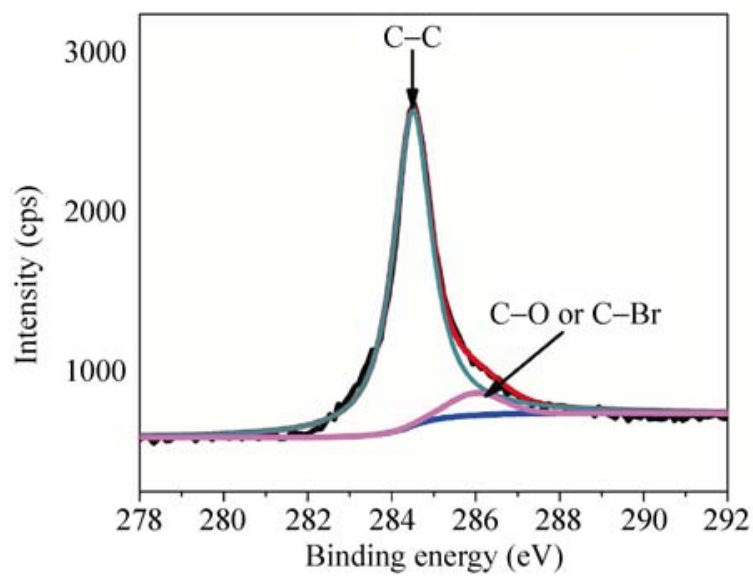

(a)

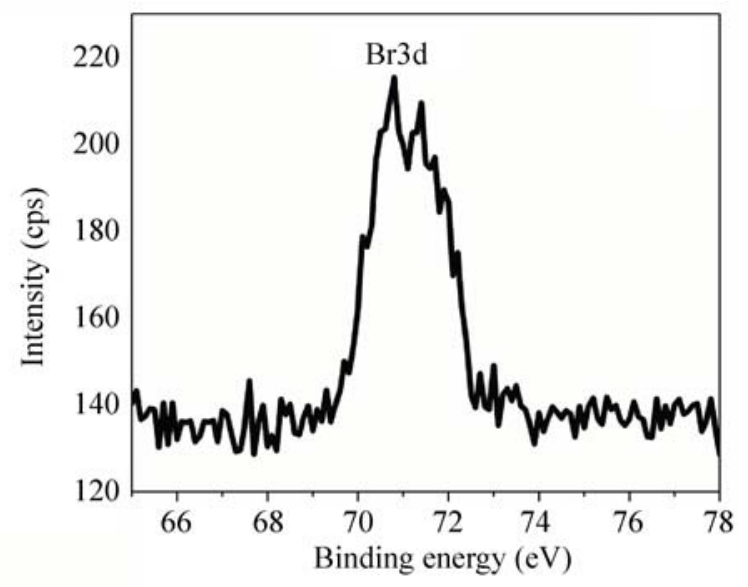

(b)

Figure 2 XPS analysis of CEG: (a) high-resolution C 1s peak (black) is fitted with $\mathrm{C}-\mathrm{C}$ peak at $284.5 \mathrm{eV}$ (green) and C-O or C-Br peak at $256 \mathrm{eV}$ (purple); (b) Br 3d peak. XPS was carried out on a PHI quantera SXM scanning X-ray microprobe with a base pressure of $5 \times 10^{-9}$ torr, with an $\mathrm{Al}$ cathode as the X-ray source set at $100 \mathrm{~W}$ and a pass energy of $140.00 \mathrm{eV}$ (survey scan) and $26.00 \mathrm{eV}$ (high-resolution scan), $45^{\circ}$ takeoff angle, and a $100-\mu \mathrm{m}$ beam size 
by normal-incidence selected area electron diffraction (SAED) labeled with the Miller-Bravais ( $h k i l$ ) indices, as shown in Fig. 3. Figure 3(a) shows a clear hexagonal electron diffraction pattern taken from a single-layer CEG. For single-layer graphene, the $\{2110\}$ spots appear to be less intense relative to the $\{1100\}$ spots [8]. The SAED of two stacked CEG layers with a twist angle between the layers is shown in Fig. 3(b). All CEG samples show a typical $A B$ stacking structure.

Analysis by high resolution transmission electron microscopy (HRTEM, JEOL 2100F field emission gun transmission electron microscope) was used to estimate the thickness of the CEG by focusing the HRTEM on the edge of the sheets. In Fig. 4, all four samples have fewer than 10 layers, which is consistent with the definition of graphene, where $<10$ layers is termed

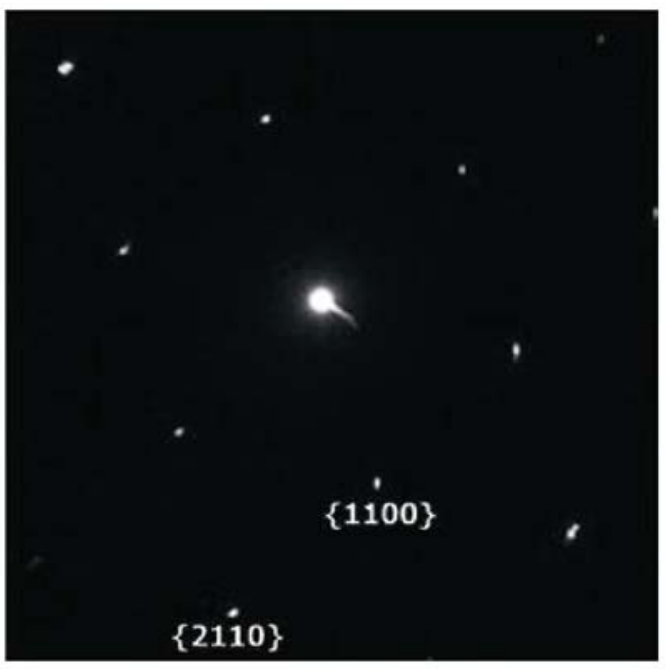

(a)

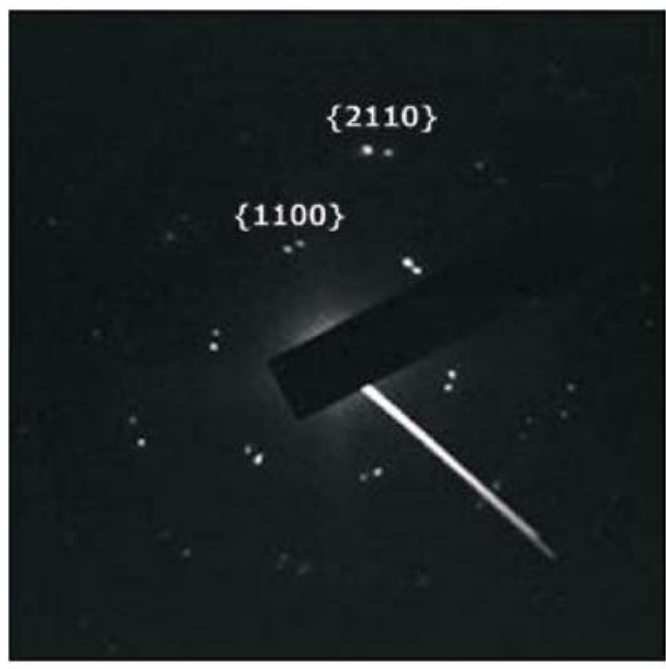

(b)

Figure 3 Selected area electron diffraction (SAED) of (a) a single layer of CEG, and (b) two single-layer CEG flakes stacked with a slight twist angle. The ratios of the intensities of the $\{2110\}$ and $\{1100\}$ diffraction peaks are $<1$, which is a signature of graphene
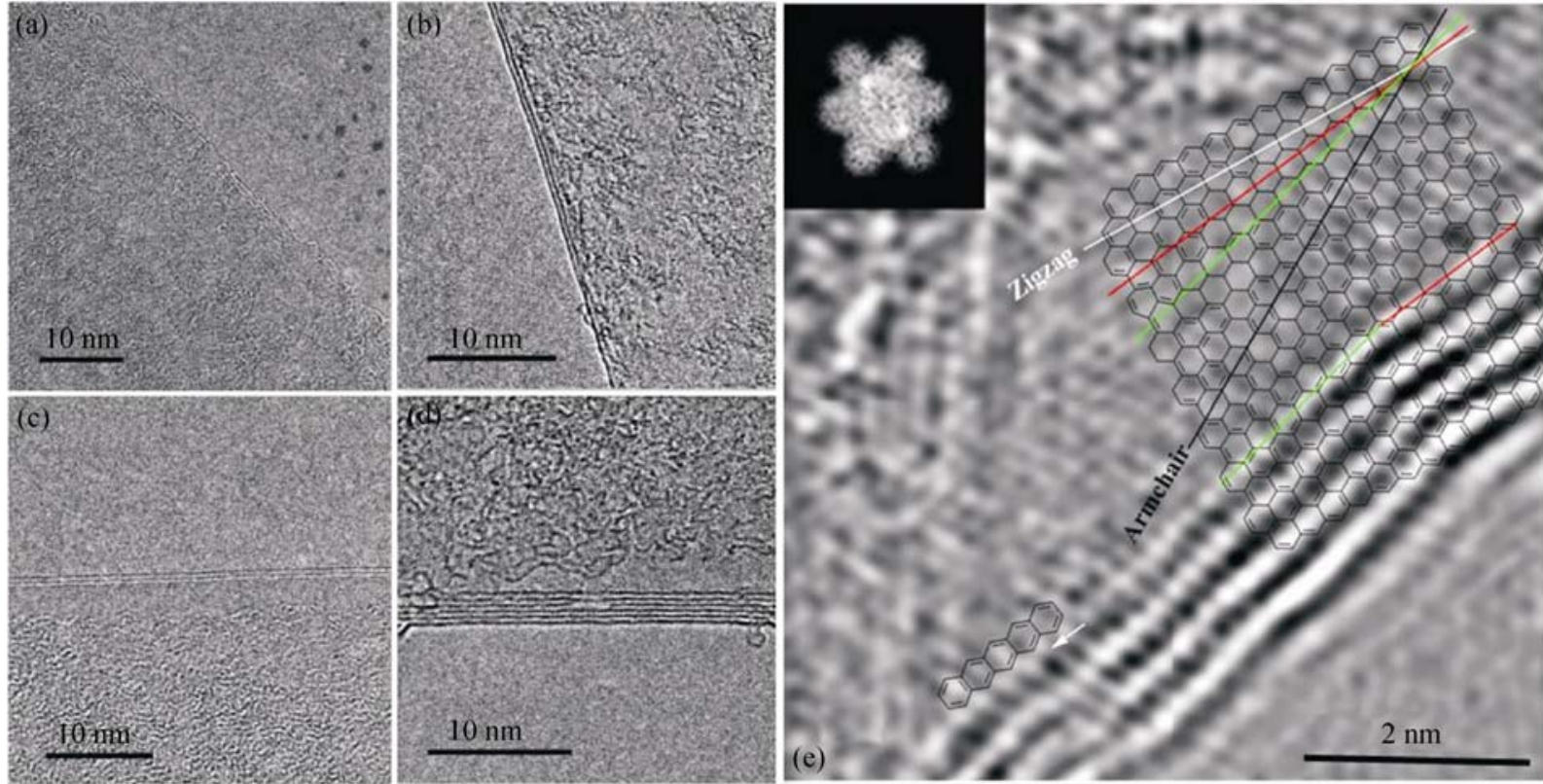

Figure 4 HRTEM of the edges of various CEG samples: (a) single-layer CEG, (b) two-layer CEG, (c) three-layer CEG, (d) six-layer CEG, and (e) simulated four-layer CEG edge (inset is the FFT) overlaid on an HRTEM. The white line is the zigzag direction while the black line shows the armchair direction. The red and green lines indicate the direction of real edges 
"graphene" [1]. Single-layer graphene must be imaged carefully since electron irradiation can easily generate defects $\left(\mathrm{sp}^{3} \mathrm{C}\right)$ in the graphene sheet [16]. The treated fast Fourier transform (FFT) HRTEM image (Fig. 4(e)) displays the lattice structure of a four-layer CEG overlaid with a polycyclic aromatic hydrocarbon model. CEG has fewer defects than graphene oxide and chemically converted graphene [17], and thus the polycyclic aromatic hydrocarbon model is a viable simulation of the structure. Crystalline graphene edges can be assigned as having either zigzag or armchair configurations [18, 19]. Energetically, zigzag edges are more stable than armchair edges [18]. Therefore, the reactivity sequence of edge $C$ atoms should be: dangling $\mathrm{C}>$ armchair $\mathrm{C}>$ zigzag $\mathrm{C}$. The edge structure was also simulated and overlaid on the image. As shown in Fig. 4(e), the white and black lines represent zigzag and armchair directions, respectively. The real edges are marked with red and green lines. However, based on the HRTEM results, the CEG edges analyzed are neither strictly zigzag nor armchair, except for a few small zigzag fragments.

There are several reasons for problems with the graphene edge simulation. First, the simulated edge may not be the real edge of the CEG, since functional groups and heteroatoms attached directly to the edges would blur the graphene boundary. It is difficult to differentiate functional groups from the graphene backbone by ordinary HRTEM. Secondly, defects and functional groups on graphene determine its surface profile. Vacancies and $\mathrm{sp}^{3}$ bonds interrupt the conjugation and make the graphene surface buckle over short ranges. This is different from long-range roughness due to the intrinsic flexibility of single graphene sheets [20], which can be overcome by tilting and focusing on a small area. Lastly, short-range surface fluctuation, especially curvature around the edge, will change the direction of the edge line and make its edge structure unpredictable. Single-layer and few-layer graphene sheets are sensitive to their chemical and physical environment, and form ripples on the nmscale. However, the simulated model is ideally flat and does not take into account the effects of rippling on the edge configuration. As shown in Fig. 4(e), the edge of the graphene sheet is pointing out of the plane and appears to be rippled.

The Raman spectrum of the expanded graphite, as shown in Fig. 5(a), has a weak D band around $1375 \mathrm{~cm}^{-1}$ and relatively strong $G$ and $2 \mathrm{D}$ bands at 1585 and $2700 \mathrm{~cm}^{-1}$, respectively. The $\mathrm{D}$ band could be ascribed to defects induced by thermal expansion and edge functionalization [21]. After the diazonium reaction, the $G$ and $2 D$ peaks in the spectrum of bulk CEG films (Fig. 5(b)) kept their intensity and shape, which indicates that the quality of graphene was largely preserved. The D band increased slightly in size, presumably because the CEG contained more functional groups than the expanded graphite. Compared to graphene oxide or chemically converted graphene, which have larger D/G ratios [15], the CEG has a relatively small $\mathrm{D} / \mathrm{G}$ ratio of $\sim 0.1$. This is consistent with the XPS results in suggesting that the CEG was lightly functionalized.

The electrical properties were measured on a CEG device built by e-beam lithography (GEOL 6500) using a Pt two-probe method. The sheet resistance was about $560 \Omega /$ sq (see Fig. S-1 in the Electronic Supplementary Material (ESM)). The high conductivity shown by the CEG device was indicative of a material with few defects in the basal plane [8-10].

\subsection{Edge selectivity of functionalization}

One multi-layered expanded graphite flake has only

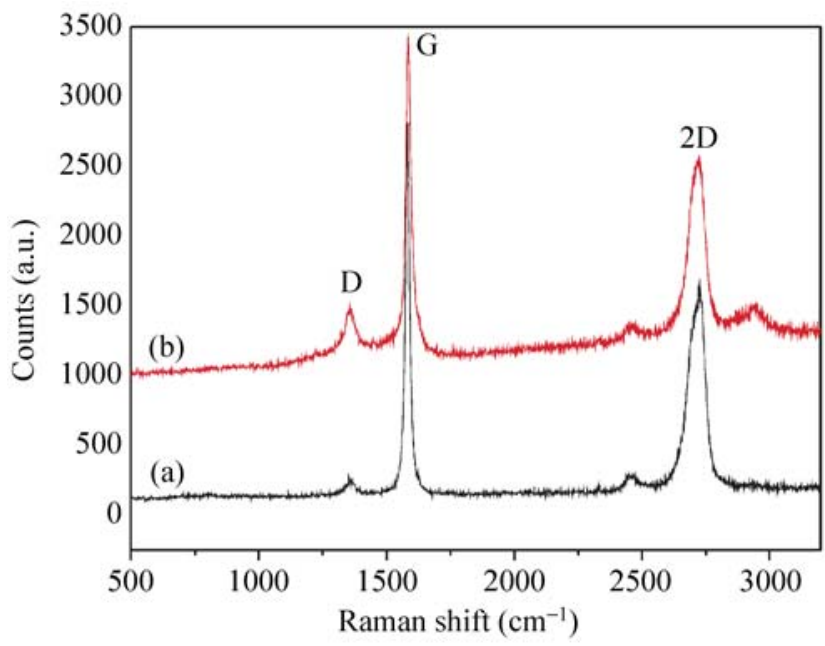

Figure 5 (a) Raman spectrum of the expanded graphite before functionalization. (b) Raman spectrum of bulk CEG films. Spectra were obtained on a Renishaw Raman scope at $514 \mathrm{~nm}$ Ar ion laser excitation at a laser power of $5 \%$ on a dry solid sample 
two exposed faces. Except for their edges, functionalization of the faces of the inner sheets is retarded since the reagents cannot readily gain access to the spaces between the sheets. That the CEG sheets have more functional groups around their edges can be confirmed by electron energy loss spectroscopy (EELS). Using elemental mapping under the EFTEM mode, the distribution of $\mathrm{Br}$ substituents can be directly obtained from the transmission electron microscopy (TEM) image. Figure 6(a) shows the zero loss TEM image of CEG. Figures 6(b) and 6(c) are the mapping of the $\mathrm{C}$ (K edge at $284 \mathrm{eV})$ and $\mathrm{Br}\left(\mathrm{M}_{45}\right.$ edge at $\left.69 \mathrm{eV}\right)$ elements present in the CEG flake. Therefore, based on the EFTEM images, the 4-bromophenyl functional groups are, in large part, bonded within $\sim 70 \mathrm{~nm}$ of the edges of the CEG. It is this region that produces most of the Raman D signal [22]. Because the thermal expansion process opens the expanded graphite edges to a certain extent, the functionalization reagents penetrate into that limited depth and graft on those near-edge planes. The edge selectivity is directly related to the degree of exposure of the interior basal planes to the functionalization reagent.

\subsection{Efficiency of chemically-assisted exfoliation}

The solubility of graphene could be increased by adding an external stabilizer. For example, $0.015 \mathrm{mg} / \mathrm{mL}$ expanded graphite can be dispersed in DMF with excess tetrabutylammonium hydroxide (TBAH) [23]. For CEG, with only a few functional groups on the edge, its solubility varies from 0.01 to $0.02 \mathrm{mg} / \mathrm{mL}$ in $\mathrm{DMF}$, without the need for exogenous stabilizers. Due to graphene's large surface area and flexible planes, when large flakes were prepared for TEM by pipetting the CEG solution onto a 300 mesh lacey carbon grid, the flakes stacked or folded. Therefore, the TEM analysis was performed only on small flakes to estimate the number of layers per flake [8]. The TEMgenerated histogram (Fig. 7 ) shows that $>70 \%$ of flakes have $<5$ layers and about $10 \%$ are single- sheet. This is comparable with the liquid phase exfoliation method [24], which reported $>63 \%$ of flakes with $1-5$ layers.

\subsection{Morphology of CEG}

The CEG sample was deposited directly on a lacey carbon TEM grid for scanning electron microscope (SEM) analysis. According to the SEM data (Fig. 8), the CEG flakes usually have a size around $1 \mu \mathrm{m}$. Some larger flakes could be visualized under an optical microscope on a 300-nm-thick layer of $\mathrm{SiO}_{2}$ atop a $\mathrm{Si}$ wafer (Fig. S-2 in the ESM). These flakes were noted in the final solution even after centrifugation. They are likely to have more functional groups that stabilize them in the DMF. The thickness of the CEG flakes was measured by atomic force microscopy (AFM, Digital Instruments Nanoscope III A, in tapping mode). Figure 9 shows a height mode scan of a CEG solution spin-coated on mica. With a height of $1.7 \mathrm{~nm}$ in Fig. 9(a) and $1.4 \mathrm{~nm}$ in Fig. 9(b), the CEG shows a
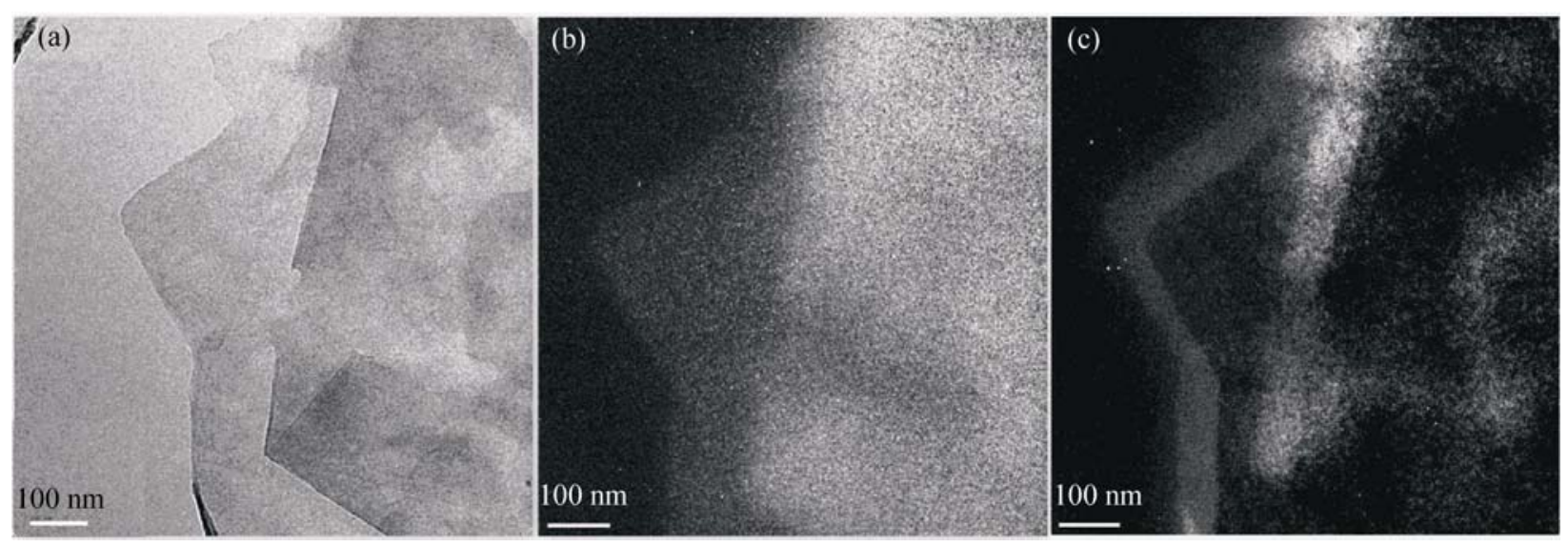

Figure 6 EFTEM of CEG flakes: (a) zero loss image, (b) C mapping (C K edge at $284 \mathrm{eV}$ ), and (c) $\mathrm{Br}$ mapping (Br $\mathrm{M}_{45} \mathrm{edge}$ at $\left.69 \mathrm{eV}\right)$. Note the elemental highlights near the edges. $\mathrm{Br}$ is only observed within $\sim 70 \mathrm{~nm}$ of the edge 


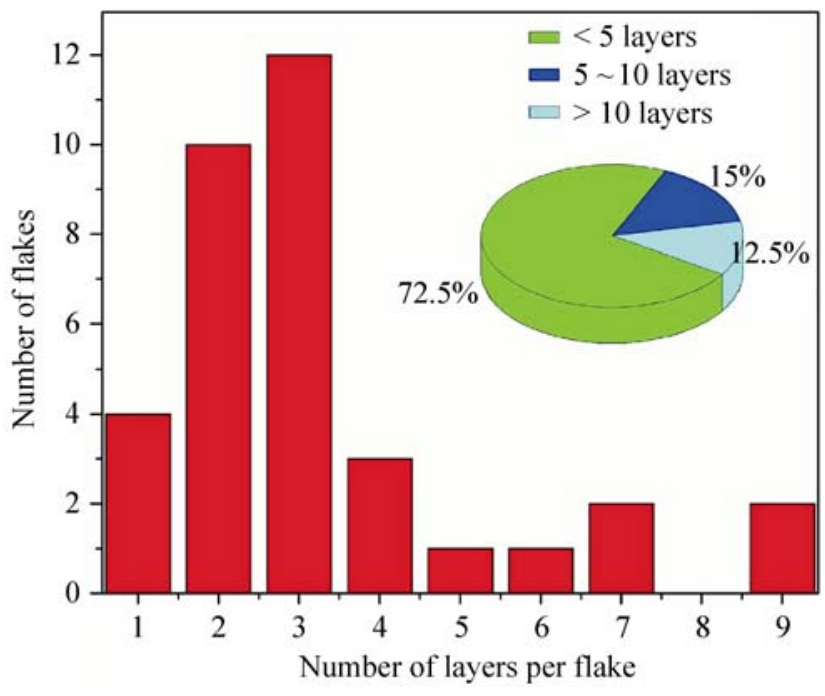

Figure 7 Histogram of number of layers per CEG flakes counted by TEM over a commonly characteristic domain of study

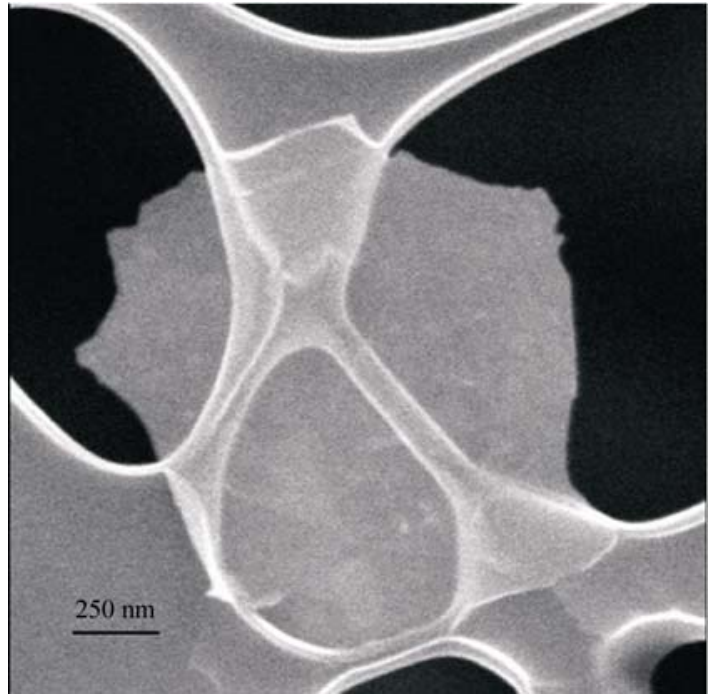

Figure 8 SEM image of CEG flake atop a lacey carbon grid

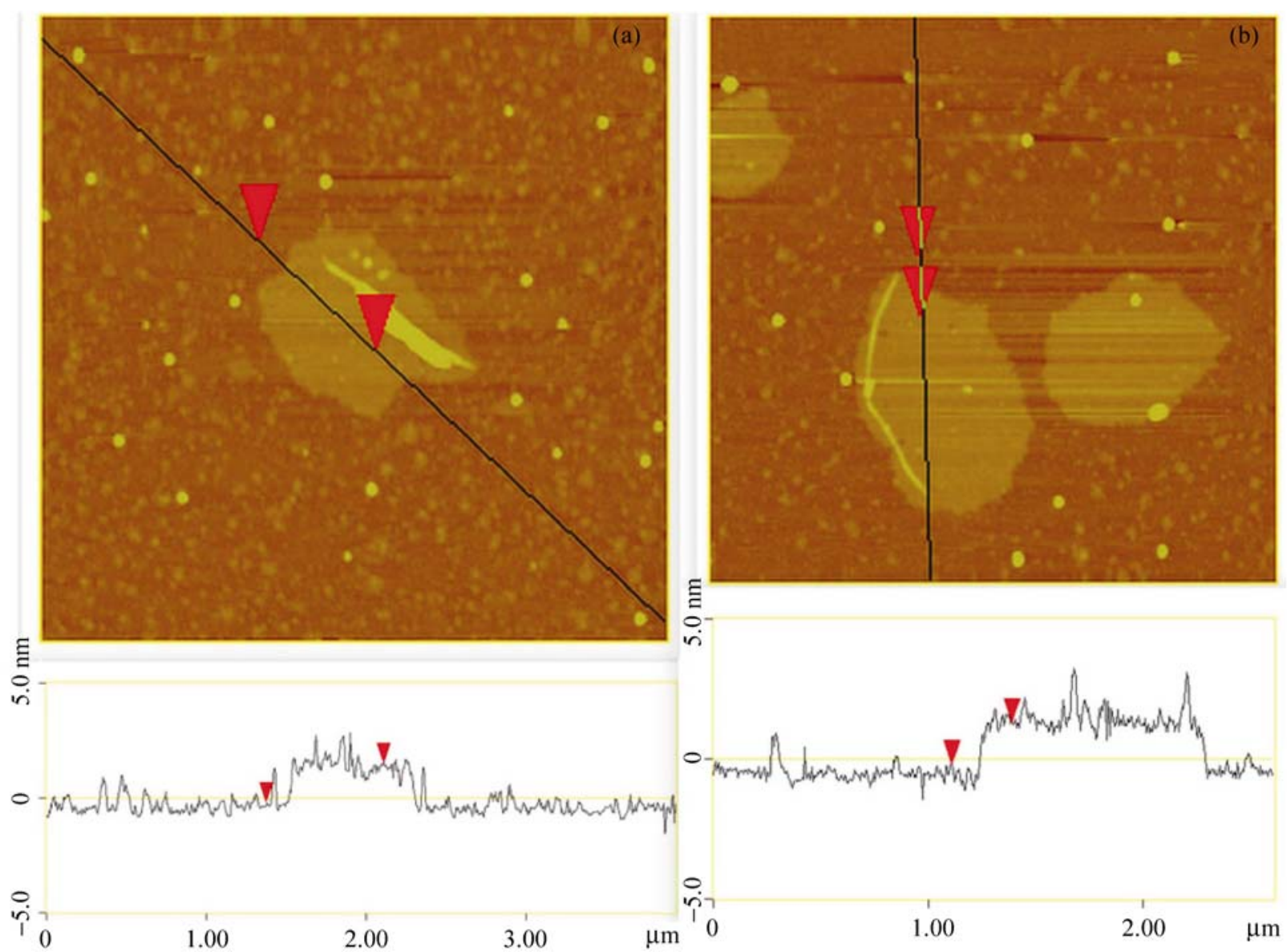

Figure 9 AFM of CEG on mica: (a) the edge of the image is $4 \mu \mathrm{m}$ and the vertical distance marked is $1.7 \mathrm{~nm}$; (b) the edge of the image is $2.5 \mu \mathrm{m}$ and the vertical distance is $1.4 \mathrm{~nm}$. The bottom plots show the height profiles along the black imaged lines above 
thickness of 1 to 2 layers, which corresponds well to the TEM measurements.

\section{Conclusions}

Expanded graphite can be converted via functionalization to CEG that is stable in DMF solvent without any added stabilizer. As established by EFTEM elemental mapping, these CEG sheets have functional groups predominantly bonded within $70 \mathrm{~nm}$ of the edges and retain the pristine graphene structure in the interior basal planes. Because of CEG's unique structure, its solubility is higher than that of pure graphene, but CEG has no need for the addition of surfactants to increase its solubility. The microscopy data indicates that more than $70 \%$ of the CEG flakes are $<5$ layers thick. In addition, the CEG preparation method is simpler than the multi-step method needed for preparing graphene oxide followed by reduction to chemically-converted graphene. The process for preparing CEG is a promising method to achieve a high yield of soluble graphene.

\section{Acknowledgements}

We thank the U.S. Department of Energy's Office of Energy Efficiency and Renewable Energy within the Hydrogen Sorption Center of Excellence, No. DE-FC36-05GO15073, the Air Force Office of Scientific Research (CONTACT), the Office of Naval Research through a Multidisciplinary University Research Initiative (MURI) with the University of California, Berkeley (00006766), and the Advanced Energy Consortium (member companies include BP America Inc., Baker Hughes Inc., Conoco-Phillips, Halliburton Energy Services Inc., Marathon Oil Corp., Occidental Oil and Gas, Petrobras, Schlumberger, Shell, and Total) for financial support. Thanks to Dr. Wenhua Guo for assistance with the TEM.

Electronic Supplementary Materials: Detailed experiment procedures, morphology characterization with SEM and optical microscope, and electrical property measurements are available in the online version of this article at http://dx.doi.org/10.1007/s12274-010-1016-2 and are accessible free of charge.

\section{References}

[1] Geim, A. K.; Novoselov, K. S. The rise of graphene. Nat. Mater. 2007, 6, 183-191.

[2] Ruoff, R. Graphene: Calling all chemists. Nat. Nanotechnol. 2008, 3, 10-11.

[3] Stankovich, S.; Dikin, D. A.; Piner, R. D.; Kohlhaas, K. A.; Kleinhammes, A.; Jia, Y. Y.; Wu, Y.; Nguyen, S. T.; Ruoff, R. S. Synthesis of graphene-based nanosheets via chemical reduction of exfoliated graphene oxide. Carbon 2007, 45, 1558-1565.

[4] Wang, H. L.; Robinson, J. T.; Li, X. L.; Dai, H. J. Solvothermal reduction of chemically exfoliated graphene sheets. J. Am. Chem. Soc. 2009, 131, 9910-9911.

[5] Novoselov, K. S.; Geim, A. K.; Morozov, S. V.; Jiang, D.; Zhang, Y.; Dubonos, S. V.; Grigorieva, I. V.; Firsov, A. A. Electric field effect in atomically thin carbon films. Science 2004, 306, 666-669.

[6] Hamilton, C. E.; Lomeda, J. R.; Sun, Z. Z.; Tour, J. M.; Barron, A. R. High-yield organic dispersions of unfunctionalized graphene. Nano Lett. 2009, 9, 3460-3462.

[7] Hernandez, Y.; Nicolosi, V.; Lotya, M.; Blighe, F. M.; Sun, Z. Y.; De, S.; McGovern, I. T.; Holland, B.; Byrne, M.; Gun'ko, Y. K.; Boland, J. J.; Niraj, P.; Duesberg, G.; Krishnamurthy, S.; Goodhue, R.; Hutchison, J.; Scardaci, V.; Ferrari, A. C.; Coleman, J. N. High-yield production of graphene by liquid-phase exfoliation of graphite. Nat. Nanotechnol. 2008, 3, 563-568.

[8] Lotya, M.; Hernandez, Y.; King, P. J.; Smith, R. J.; Nicolosi, V.; Karlsson, L. S.; Blighe, F. M.; De, S.; Wang, Z.; McGovern, I. T.; Duesberg, G. S.; Coleman, J. N. Liquid phase production of graphene by exfoliation of graphite in surfactant/water solutions. J. Am. Chem. Soc. 2009, 131, 3611-3620.

[9] Reina, A.; Jia, X. T.; Ho, J.; Nezich, D.; Son, H. B.; Bulovic, V.; Dresselhaus, M. S.; Kong, J. Large area, few-layer graphene films on arbitrary substrates by chemical vapor deposition. Nano Lett. 2009, 9, 30-35.

[10] Jia, X. T.; Hofmann, M.; Meunier, V.; Sumpter, B. G.; Campos-Delgado, J.; Romo-Herrera, J. M.; Son, H. B.; Hsieh, Y. P.; Reina, A.; Kong, J.; Terrones, M.; Dresselhaus, M. S. Controlled formation of sharp zigzag and armchair edges in graphitic nanoribbons. Science 2009, 323, 1701-1705. 
[11] Li, X. S.; Cai, W. W.; An, J. H.; Kim, S.; Nah, J.; Yang, D. X.; Piner, R.; Velamakanni, A.; Jung, I.; Tutuc, E.; Banerjee, S. K.; Colombo, L.; Ruoff, R. S. Large-area synthesis of high-quality and uniform graphene films on copper foils. Science 2009, 324, 1312-1314.

[12] Robinson, J. A.; Puls, C. P.; Staley, N. E.; Stitt, J. P.; Fanton, M. A.; Emtsev, K. V.; Seyller, T.; Liu, Y. Raman topography and strain uniformity of large-area epitaxial graphene. Nano Lett. 2009, 9, 964-968.

[13] Ni, Z. H.; Chen, W.; Fan, X. F.; Kuo, J. L.; Yu, T.; Wee, A. T. S.; Shen, Z. X. Raman spectroscopy of epitaxial graphene on a SiC substrate. Phys. Rev. B. 2008, 77, 115416.

[14] Rohrl, J.; Hundhausen, M.; Emtsev, K. V.; Seyller, T.; Graupner, R.; Ley, L. Raman spectra of epitaxial graphene on SiC(0001). Appl. Phys. Lett. 2008, 92, 201918.

[15] Lomeda, J. R.; Doyle, C. D.; Kosynkin, D. V.; Hwang, W. F.; Tour, J. M. Diazonium functionalization of surfactantwrapped chemically converted graphene sheets. J. Am. Chem. Soc. 2008, 130, 16201-16206.

[16] Teweldebrhan, D.; Balandin, A. A. Modification of graphene properties due to electron-beam irradiation. Appl. Phys. Lett. 2009, 94, 013101.

[17] Mkhoyan, K. A.; Contryman, A. W.; Silcox, J.; Stewart, D. A.; Eda, G.; Mattevi, C.; Miller, S.; Chhowalla, M. Atomic and electronic structure of graphene-oxide. Nano Lett. 2009, 9, 1058-1063.
[18] Girit, C. O.; Meyer, J. C.; Erni, R.; Rossell, M. D.; Kisielowski, C.; Yang, L.; Park, C. H.; Crommie, M. F.; Cohen, M. L.; Louie, S. G.; Zettl, A. Graphene at the edge: Stability and dynamics. Science 2009, 323, 1705-1708.

[19] Liu, Z.; Suenaga, K.; Harris, P. J. F.; Iijima, S. Open and closed edges of graphene layers. Phys. Rev. Lett. 2009, 102, 015501 .

[20] Barnard, A. S.; Snook, I. K. Thermal stability of graphene edge structure and graphene nanoflakes. J. Chem. Phys. 2008, 128, 094707.

[21] Pimenta, M. A.; Dresselhaus, G.; Dresselhaus, M. S.; Cancado, L. G.; Jorio, A.; Saito, R. Studying disorder in graphite-based systems by Raman spectroscopy. Phys. Chem. Chem. Phys. 2007, 9, 1276-1291.

[22] Gupta, A. K.; Russin, T. J.; Gutierrez, H. R.; Eklund, P. C. Probing graphene edges via Raman scattering. ACS Nano 2009, 3, 45-52.

[23] Li, X. L.; Zhang, G. Y.; Bai, X. D.; Sun, X. M.; Wang, X. R.; Wang, E.; Dai, H. J. Highly conducting graphene sheets and Langmuir-Blodgett films. Nat. Nanotechnol. 2008, 3, 538-542.

[24] Hernandez, Y.; Lotya, M.; Rickard, D.; Bergin, S. D.; Coleman, J. N. Measurement of multicomponent solubility parameters for graphene facilities solvent discovery. Langmuir, in press, DOI: 10.1021/la903188a. 